\title{
The time course of individual face recognition: a pattern analysis of ERP signals
}

\author{
Dan Nemrodov ${ }^{*}$, \\ Matthias Niemeier ${ }^{1}$ \\ Jenkin Ngo Yin $\mathrm{Mok}^{2}$, \\ Adrian Nestor ${ }^{1}$
}

${ }^{1}$ Department of Psychology at Scarborough, University of Toronto, 1265 Military Trail,
Toronto, Ontario, Canada
${ }^{2}$ Department of Psychology, York University, 4700 Keele Street, Toronto, Ontario, Canada
* To whom correspondence should be addressed.
E-Mail : dan.nemrodov@utoronto.ca

Tel : +1 (647) 772-0047 


\begin{abstract}
An extensive body of work documents the time course of neural face processing in the human visual cortex. However, the majority of this work has focused on specific temporal landmarks, such as N170 and N250 components, derived through univariate analyses of EEG data. Here, we take on a broader evaluation of ERP signals related to individual face recognition as we attempt to move beyond the leading theoretical and methodological framework through the application of pattern analysis to ERP data. Specifically, we investigate the spatiotemporal profile of identity recognition across variation in emotional expression. To this end, we apply pattern classification to ERP signals both in time, for any single electrode, and in space, across multiple electrodes. Our results confirm the significance of traditional ERP components in face processing. At the same time though, they support the idea that the temporal profile of face recognition is incompletely described by such components. First, we show that signals associated with different facial identities can be discriminated from each other outside the scope of these components, as early as $70 \mathrm{~ms}$ following stimulus presentation. Next, electrodes associated with traditional ERP components as well as, critically, those not associated with such components are shown to contribute information to stimulus discriminability. And last, the levels of ERP-based pattern discrimination are found to correlate with recognition accuracy across subjects confirming the relevance of these methods for bridging brain and behavior data. Altogether, the current results shed new light on the fine-grained time course of neural face processing and showcase the value of novel methods for pattern analysis to investigating fundamental aspects of visual recognition.
\end{abstract}

Keywords ERP, visual face recognition, pattern analysis, N170, P1, time course. 


\section{Introduction}

Elucidating the time course of individual face recognition has been the focus of extensive research in the study of face recognition and of its neural underpinnings. Electroencephalography (EEG) and magnetoencephalography (MEG), in particular, have provided valuable tools in addressing this challenge due to their superior temporal resolution. Their application to the study of face processing has established the existence of robust temporal landmarks such as the facerelated N170 component. However, the perceptual significance of these landmarks, such as their relevance for individual or general face recognition, remains a topic of considerable controversy (for reviews see Eimer, 2011; Rossion and Jacques, 2011). Here we address this issue with the use of pattern analysis techniques as applied to ERP responses associated with face processing. Theoretically, the current work aims to shed new light on a fundamental aspect of neural face processing, that is, the temporal profile of individual face recognition, while, methodologically, it speaks to the informational content of EEG signals and to its derivation via adequate techniques.

How quickly can our visual system extract and compute perceptual representations of facial identity? The preferential response to faces of the classical N170 ERP component (Bentin et al., 1996; Linkenkaer-Hansen et al., 1998) and of its MEG counterpart, the M170 (Halgren et al., 2000; Liu et al., 2002), raises an obvious possibility in this respect. Specifically, it is possible that such components mark not only category-level responses to faces (versus other types of stimuli) but also identity-related responses associated with face recognition at the individual level. Several ERP studies (Caharel et al., 2009; Guillaume et al., 2009; Heisz et al., 2006; Itier and Taylor, 2002) and MEG studies (Harris and Nakayama, 2008; Liu et al., 2002) have provided support for this hypothesis. Yet, other research groups have contended that representations of 
facial identity are indexed by later components, such as the N250 or even the N400 (Bentin and Deouell, 2000; Huddy et al., 2003; Schweinberger et al., 2002; Tanaka et al., 2006; Zheng et al., 2012). At the other end, the earlier P1 component, with a peak latency around $100 \mathrm{~ms}$ after stimulus onset, has also been proposed as a marker of individual face recognition (Debruille et al., 1998) or, at least, of category-level face processing (Dering et al., 2011; Herrmann et al., 2005). However, its sensitivity to face processing, even at the category level, has been repeatedly challenged (Bentin et al., 2007; Kuefner et al., 2010; Rossion and Jacques, 2008).

Thus, despite substantial efforts dedicated to clarifying the time course of individual face recognition, a conclusive resolution of this issue has eluded the field. Several likely causes underlie the discrepancies noted above. First, regarding the nature of the stimuli used, many studies employ familiar or famous faces that engage complex semantic and emotional processes in addition to perceptual ones. Such processes may naturally emphasize later components at the expense of earlier ones. On the other hand, broad differences in low-level image properties could be misinterpreted as face-sensitive responses at early stages of perceptual processing. Second, many studies deploy delay repetition designs that may lack the power to uncover identity-related effects or, conversely, adaptation methods with short interstimulus intervals that may provide effects non-specific to targets (Nemrodov and Itier, 2012). Third, most research has focused on a handful of ERP components because of their prominence. However, the exclusive focus on such components may provide an incomplete and potentially misleading view of neural face processing (Rousselet and Pernet, 2011).

More generally, in the study of face recognition, current work with either EEG or MEG modalities relies primarily on univariate signals despite the availability of multivariable information in the spatial domain, that is, across electrodes, and especially in the temporal domain, that is, across multiple time points (but see Meng et al., 2012; Moulson et al., 2011; 
Stewart et al., 2014; for recent applications of pattern classification to EEG in related fields). Relevantly here, the use of pattern analysis in fMRI investigations of face processing (Haxby et al., 2001) has allowed considerable advancements in clarifying the neural basis of individual face recognition. For instance, several studies (Anzellotti et al., 2014; Goesaert and Op de Beeck, 2013; Kriegeskorte et al., 2007; Natu et al., 2010; Nestor et al., 2011) have shown that individual face recognition relies on a network of regions extending beyond the classical face-selective regions localized by univariate mapping. By the same token, pattern analyses of EEG signals may be able to capture sensitivity to this type of visual recognition beyond traditional ERP components as well as to shed new light on the involvement of these components in identity processing.

The current study addresses the issues above by investigating identity discrimination across variation in emotional expression from ERP signals. Specifically, we examine the ability of temporal and spatial ERP patterns to distinguish between unfamiliar individuals displaying neutral and happy expressions - to be clear, the use of the emotional expressions allows us to vary image properties in a meaningful way while keeping identity information constant. In doing so, the present work takes on three distinct goals: (a) evaluating the involvement of classical ERP components in individual face recognition; (b) exploring the time course of identity discrimination from the ERP signal, and (c) characterizing the broad spatial profile of identity discrimination. The results of our investigation support the role of traditional ERP components in identity processing. At the same time though they show that the neural markers of identity discrimination extend beyond such components and, spatially, the source of discrimination evolves over time in a posterior-to-anterior fashion. Last, methodologically, the present findings showcase the ability of pattern analysis techniques to complement conventional univariate analyses as applied to ERP signals. 


\section{Materials and Methods}

\section{Participants}

Fourteen healthy Caucasian participants (4 males, 10 females; age range: 19-24 years) with normal or corrected-to-normal vision were recruited from the University of Toronto community to participate in exchange for payment or course credit. Ten of the participants, who met the criteria stipulated below (Procedures), took part in the main study. All participants provided informed consent. The study was approved by the Research Ethics Board at University of Toronto.

\section{Stimuli}

Eight images of four individuals displaying neutral and happy expressions (Fig. 1) were used as experimental stimuli. The images were selected from a larger dataset of 240 color face images collected from the FERET database (Phillips et al., 2000, 1998), AR (Martinez and Benavente, 1998) and Radboud (Langner et al., 2010). The dataset contained images of young adult Caucasian individuals with frontal pose, frontal gaze and frontal illumination. Experimental stimuli ( 2 males and 2 females) were selected so that no facial accessories, hair or makeup obscured the internal features of the face and all happy expressions displayed an open-mouth smile. These images were further scaled with the same interocular distance, cropped to eliminate background and normalized with the same mean and root mean square (RMS) contrast values separately for each color channel in CIEL*a*b* color space.

Figure 1 


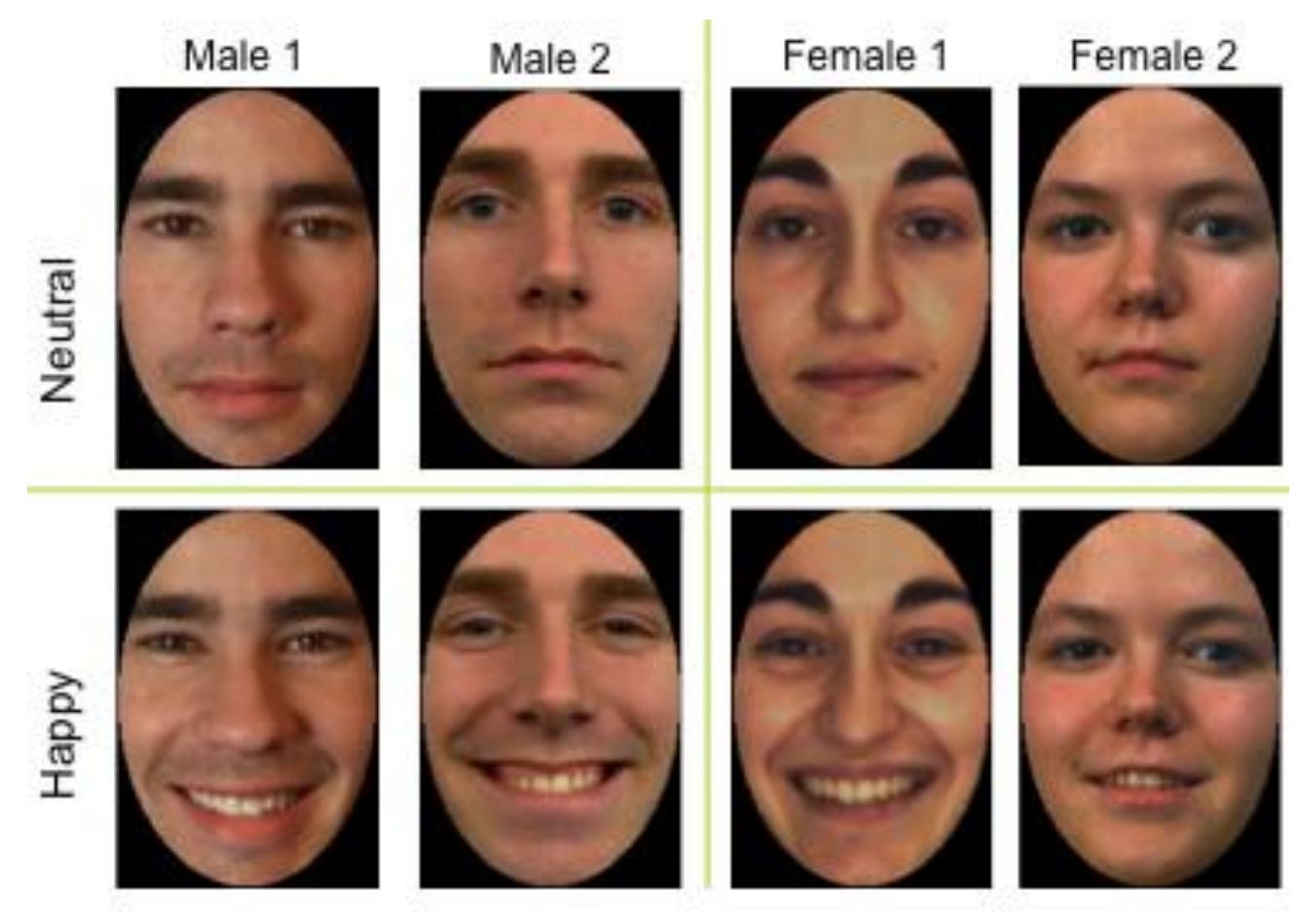

\section{Procedures}

Participants were presented with sequences of experimental stimuli and were asked to complete a one-back identity task. Specifically, each experimental block consisted of a sequence of 200 trials ( 25 trials X 8 images) containing 64 repetitions of the previously shown identity (16 repetitions $\mathrm{X} 4$ identities). Importantly, trials were pseudorandomized so that a facial identity appearing twice in a row displayed different emotional expressions. Within each trial a fixation cross was presented for a variable duration between 600 -1000 ms and was followed by a stimulus for $400 \mathrm{~ms}$. Participants were asked to press a button whenever they noticed that the same facial identity was presented twice in a row.

During the experiment, participants were seated in a dimly lit and quiet room. Stimuli were presented in the center of a CRT screen $(100 \mathrm{~Hz}$ refresh rate) against a dark background and subtended a visual angle of $1.43^{\circ} \times 2^{\circ}$ from a viewing distance of approximately $80 \mathrm{~cm}$. For any 
given stimulus, the presentation delay introduced by the raster scan nature of the projection is approximately 5ms (and all latencies reported below have been adjusted accordingly). Stimulus presentation and response recording relied on Matlab (Mathworks, Natick, MA) and Psychtoolbox 3.0.8 (Brainard, 1997; Pelli, 1997).

A behavioral pretest consisting of nine blocks was used to assess performance. Participants exhibiting recognition accuracy higher than $80 \%$ were invited to take part in the main experiment. The pretest also aimed to minimize training effects over the course of the EEG sessions by using the same task and stimuli described above.

EEG recording was carried out across 26 blocks split across two experimental sessions performed on separate days (leading to a total of 650 presentations per stimulus image). Due to technical reasons, one participant completed only 23 blocks, and two participants completed only 25 blocks. Each experimental session lasted between 1 and 1.5 hours.

\section{EEG acquisition and Preprocessing}

High-density EEG was recorded using ASA (ANT B.V., Enschede, The Netherlands) from 64 sintered $\mathrm{Ag} / \mathrm{AgCl}$ scalp electrodes mounted on an elastic cap utilizing the International 10-5 electrode system (Waveguard, ANT, and ElectrodeArrays). The electrode AFz served as ground and the left mastoid served as online reference. The reference was recomputed offline based on the average of all electrodes, excluding ocular electrodes. Electrode impedances were kept below $5 \mathrm{k} \Omega$. The EEG signal was amplified with an ANT high-density amplifier (22 bit, $71.5 \mathrm{nV} / \mathrm{bit}$ ) at a sampling rate of $512 \mathrm{~Hz}$. The data were band-pass filtered offline between 0.1 and $40 \mathrm{~Hz}$ (24 dB per octave). Eye movements were monitored with horizontal (HEOG) and vertical electrooculograms (VEOG). 
One-back repetition trials as well as false alarm trials (containing incorrect button presses) were excluded from analyses. Further, epochs with voltages exceeding $\pm 70 \mu \mathrm{V}$ at any electrode were excluded from the analysis and ocular artefacts were removed using independent component analysis (ICA) decomposition (Jung et al., 2000). The resulting number of trials retained per block for each image ranged from 15 to 23 , with an average of 21.4 trials across participants. In each block, epochs ranging from $-100 \mathrm{~ms}$ to $700 \mathrm{~ms}$ relative to stimulus onset, with the prestimulus period ( $-100 \mathrm{~ms}$ to $0 \mathrm{~ms})$ serving as baseline, were extracted and averaged to create ERPs.

\section{Conventional ERP Analyses}

To perform a coarse test for the presence of identity-related information within the ERP data, a single ERP trace was constructed through averaging across all blocks separately for each facial identity. Electrodes with maximum N170 amplitudes were identified at right $(\mathrm{P} 8, \mathrm{PO} 8)$ and left (P7, PO7) occipitotemporal (OT) sites. Then, for each participant and for each electrode we separately identified three classical ERP components potentially involved in individual face recognition: P1 (within the range of 90-130 ms), N170 (150-210 ms) and N250 (220-310 ms). These components were characterized in terms of maximum amplitude and latency-to-peak. Both measurements were subjected to a two-way ANOVA across participants - laterality (left: PO7, P7; and right: $\mathrm{P} 8, \mathrm{PO} 8) \mathrm{X}$ identity (4 facial identities).

Next, a more thorough version of the analysis above considered identity-related effects at all time points during an epoch. Concretely, instead of targeting specific components, we subjected ERP amplitudes to a sequence of one-way ANOVAs across participants, separately for each time point and each electrode. The results of these analyses were then corrected for multiple 
comparisons using the false discovery rate (FDR) (Benjamini and Hochberg, 1995; for its application to EEG see Causeur et al., 2012).

Last, a classification-based analogue of the analysis above was conducted in order to facilitate the comparison between conventional univariate analyses and pattern-based analyses of ERP signals. Of note, in this case block-specific ERPs were not averaged but, instead, provided separate observations for classification purposes (for more information regarding this analysis see Supplementary Material).

\section{Pattern classification of ERP signals - main approach}

Considering the possibility that identity-related information resides in multivariable patterns across electrodes and/or across time points it is likely that such information would be underused by conventional univariate analyses - for instance, this information could be degraded or missed as a result of variable selection (e.g., while targeting exclusively ERP components) or through averaging procedures (e.g., across multiple electrodes). Hence, a suitable patternclassification approach able to exploit such information may shed new light on the spatial profile of identity recognition.

To investigate the possibility above, pattern classification was conducted across electrodes with the use of linear Support Vector Machine (SVM) classifiers. Our choice was motivated in this respect by SVM's robust approach to classification as illustrated, for instance, by their ability to scale well with increases in pattern dimensionality (Misaki et al., 2010). To this end the data were processed according to the following procedures.

First, ERP traces corresponding to each image were averaged within blocks and convolved with a Gaussian kernel having a $23 \mathrm{~ms}$ full width at half maximum (FWHM) in order 
to reduce temporal noise in EEG-based classification patterns - the use of alternative kernels (e.g., with 14ms and 47ms FWHM) produced roughly similar classification results.

Second, ERP traces were normalized across all images and across all blocks by z-scoring data separately for each time point and each electrode. Extreme values (i.e., farther than 3 standard deviations from the mean) were thresholded and replaced with corresponding alternative values (i.e., \pm 3 ) so as to reduce their impact on SVM-based classification. Further, the data was rescaled within the interval 0-1 to facilitate its processing with the LIBSVM library for pattern classification (Chang and Lin, 2011).

Third, patterns of normalized signals across all electrodes were subjected to SVM classification (penalty parameter $c=1$ ), separately at each time point. One-against-one classification was conducted for each pair of facial identities using a leave-one-block-out crossvalidation schema. For instance, all 50 patterns corresponding to the two male faces displaying a neutral expression from blocks 1-25 were used to train a classifier. Then, the performance of the classifier was tested using the traces of the two males displaying a happy expression from the left-out block (i.e., block 26 in this case). Further, this procedure was conducted by leaving out systematically any single block and, also, by reversing the role of the two expressions (i.e., happy expressions were used for training and neutral ones were used for testing purposes).

Next, discrimination performance was encoded as sensitivity (d') at each time point for each pair of identities. Considering the fact that the discrimination of faces of different genders may benefit from additional visual and semantic cues over that of faces of the same gender, sensitivity estimates were averaged and assessed separately within and across gender (i.e., two same-gender identity pairs and four different-gender identity pairs). 
Last, the classification procedure above was conducted separately for each participant and statistical significance was assessed by comparing discrimination performance to chance $\left(d^{\prime}=0\right)$ across participants (one-sample two-tailed t-tests) separately at each time point - the results were corrected for multiple comparisons across time points using FDR (the correction was performed across time points and further restricted to temporal intervals of at least 10ms).

Importantly, the procedure above aims to preclude dependency between training and testing data (by using data from separate blocks) as well as to minimize the contribution of lowlevel visual cues to classification (by training and testing on images with different expressions). Also, one-against-one classification for each pair of faces is carried out (instead of one-againstall) so as to allow teasing apart identity-related effects within gender from those across gender.

\section{Pattern classification of ERP signals - additional analyses}

While pattern discrimination was evaluated above at the group level with the aid of parametric tests, it is informative to establish whether the outcome of these analyses are robust enough to be obtained even within single individuals with the help of nonparametric statistics. To this end, whole-array SVM-based discrimination in single subjects was compared against chance via a permutation test separately at each time point. Specifically, the labels (i.e., identity) of each observation (i.e., electrode pattern) were randomly permuted prior to classification and chance performance was evaluated based on a total of $10^{4}$ permutations for each pair of identities. Then, the actual performance, relying on the correct matching of labels with observations, was compared against chance estimates through a two-tailed permutation test.

Further, in order to assess the relationship between neural-based discrimination and behavioral performance, whole-array SVM-based discrimination was correlated with the 
behavioral accuracy of each subject averaged across all experimental blocks. Specifically, recognition accuracy was compared with neural estimates of discrimination at each time point across subjects via Pearson correlation.

Last, all the pattern analyses reported above consider spatial information across multiple electrodes at each time point with the aim of revealing the temporal profile of individual face recognition. A homologous approach involves the classification of temporal patterns separately for each electrode with the aim of revealing the spatial profile of identity recognition. This complementary approach was employed twice. First, SVM-based discrimination was computed using entire post-stimulus ERP traces associated with different identities. And, second, the analysis was restricted to distinct consecutive windows of $50 \mathrm{~ms}$ to reveal a more comprehensive spatiotemporal profile of individual face recognition

All results reported above were corrected for multiple comparisons using FDR. For analyses across spatial patterns the correction was performed, again, across time points while for analyses involving temporal or spatiotemporal patterns the correction was carried out across electrodes.

All analyses were conducted in Matlab with the help of the LIBSVM library for pattern classification (Chang and Lin, 2011).

\section{Results}

\section{Behavioral results}

Average recognition accuracy and reaction time (RT) for same-identity stimuli were 92.20\% ( $\mathrm{SD}=8 \%$ across participants) and $572 \mathrm{~ms}(\mathrm{SD}=58.30 \mathrm{~ms})$, respectively. Both accuracy and RT measures were next analyzed using two-way repeated measures ANOVA (Identity X 
Expression). The analysis of accuracy did not yield any significant effects. RT analyses showed a main effect of identity $(\mathrm{F}(3,27)=6.46, p<0.02)$ and an interaction between identity and expression $(\mathrm{F}(3,27)=3.42, p<0.032)$. Further post hoc analyses revealed that one of the female identities triggered faster responses than one of the male identities ( $<<0.001$, Bonferroni-corrected) - the two identities correspond to columns 2 and 3 in Fig 1. No other comparison was significant.

\section{The time course of identity processing - conventional univariate analyses}

Three ERP components (P1, N170 and N250) were identified separately for each participant at four OT electrodes (P8, PO8, P7, PO7). Then, mean amplitudes were assessed for each component through a two-way ANOVA across participants - laterality (left: PO7, P7; and right: P8, PO8) X identity (4 facial identities). These analyses found significant effects of identity for the $\mathrm{N} 170$ component $(\mathrm{F}(3,27)=6.075, p<0.004)$ and the $\mathrm{N} 250$ component $((\mathrm{F}(3,27)=17.614$, $p<0.001)$ but not for P1 $(p>0.558)$ - see Fig. 2. Further post hoc tests showed that one of the female identities (column 3 in Fig 1) elicited different amplitudes from one male identity (column 1 in Fig 1) for N170 ( $p=0.031$, Bonferroni-corrected) and from all other identities for N250 ( $p$ 's $<$ 0.001). No laterality effects were found but an interaction between laterality and identity was significant for $\mathrm{N} 250$ only $(\mathrm{F}(3,27)=3.535, p<0.028)$. Additional post hoc tests for this component indicated that amplitude differences between the female identity noted above and the other identities were larger on the right than on the left $(p$ 's $<0.01)$. No significant effects emerged from the analysis of component latencies.

Figure 2 

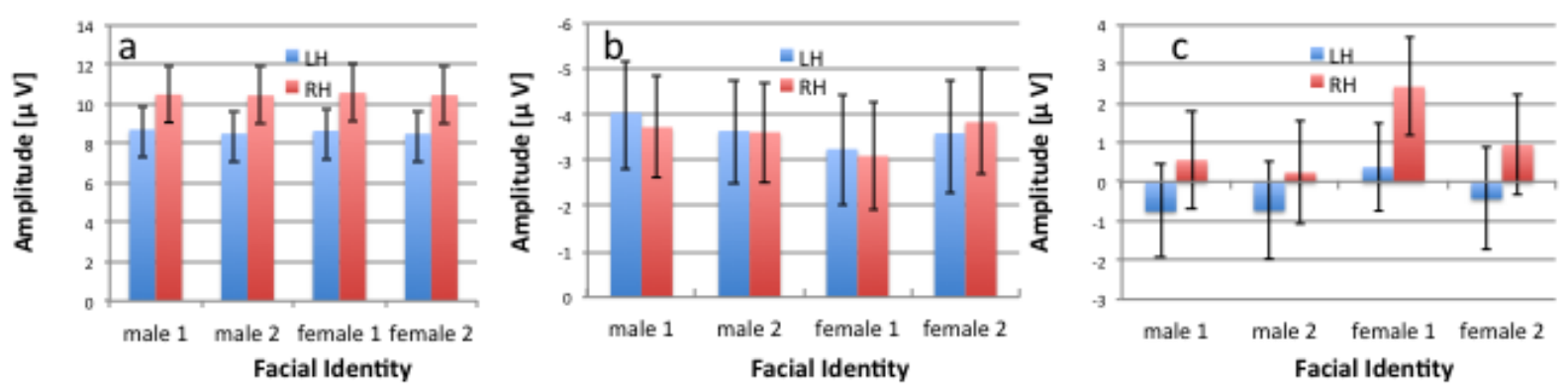

Further, a more exhaustive examination of ERP amplitudes was conducted separately for each of four OT electrodes at each time point through a series of one-way ANOVAs (across 4 facial identities). Of note, this analysis does not target individually or group-defined ERP components but, for convenience, it is limited to sites typically involved in face processing that also exhibit, in our case, the largest N170 component.

The analyses above found main effects of identity bilaterally, at P8, from $139 \mathrm{~ms}$ following stimulus presentation until the end of an epoch, and at P7, from $127 \mathrm{~ms}$ until the end, with the exception of a brief period between 184 and 215 ms (Supplementary Fig. 1). Similar results were found for P08 (from $182 \mathrm{~ms}$ until the end, with the exception of a brief period between 662 and $703 \mathrm{~ms}$ ) and for P07 (from $229 \mathrm{~ms}$ until $578 \mathrm{~ms}$ following stimulus presentation).

Overall, the two sets of results above are consistent with each other and, more generally with reports of N170 and N250 sensitivity to facial identity. Of note, the current analyses take advantage of a relatively large number of stimulus presentations to establish the coarse impact of facial identity on ERP amplitudes at designated electrodes. Their outcome serves as a starting point for our investigation by providing initial evidence for the presence of identity-related effects within the ERP signal. However, these effects may be inflated by the influence of lowlevel image differences (as the analysis only considers inter-individual but not within-individual image variability). Also, the effects reported above may be largely due to facial gender 
discrimination (as driven by faces of different genders). For this reason, further classification analyses are employed to confirm and to clarify the signature and the scope of identity processing within ERP signals.

The time course of identity discrimination - pattern classification

The temporal profile of identity discrimination was examined by the application of pattern classification to ERP amplitudes separately at each time point during an epoch. To address the issues raised by the analyses above, classification was conducted using training and test observations with different emotional expressions. Also, classification results were averaged separately for identity discrimination (i.e., for same-gender pairs) and for gender (i.e., for different-gender pairs).

SVM-based classification, as applied to the entire array of electrodes, identified two significant intervals both for identity (63-96ms; $106-800 \mathrm{~ms})$ and for gender discrimination (69 -86ms; 107-800ms). The time course for both types of discrimination is displayed in Fig. 3a for simplicity, significant intervals are only marked for identity in line with the main focus of our investigation. Further tests were also conducted to evaluate the difference between identity versus gender discrimination following pattern analysis. However, no significant intervals were found between the two. On the other hand, identity discrimination was found significant at multiple, largely overlapping intervals even when evaluating the results separately for each gender (Supplementary Fig. 2). (For a comparison of SVM-based discrimination with a classification analogue of conventional univariate analyses see supplementary material and Supplementary Fig. 3, 4.)

Figure 3 


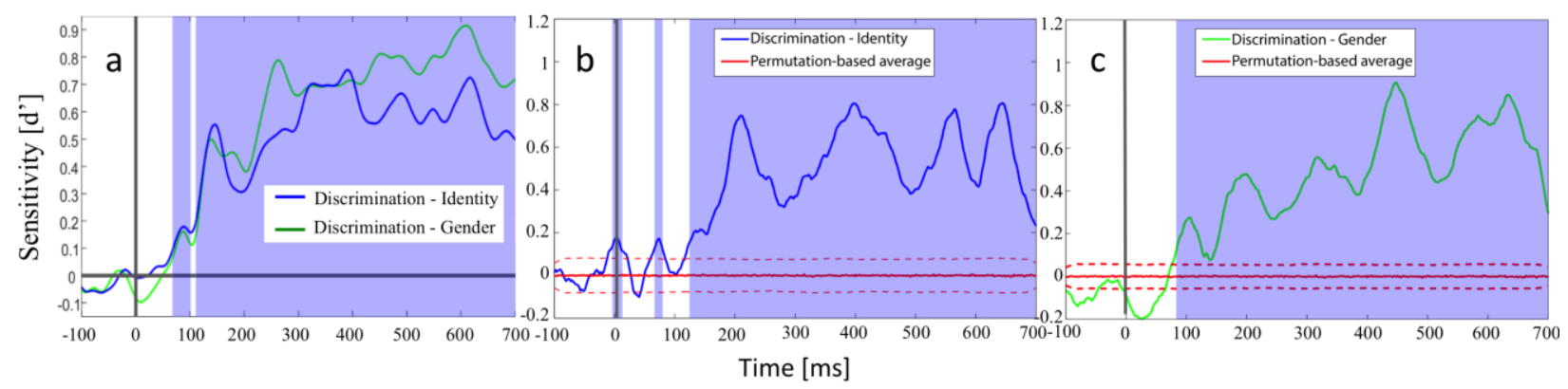

Complementing the analyses above, nonparametric tests were conducted to confirm the current results at the individual level. To this end, single-subject discrimination results were evaluated against chance by permutation tests. Overall, we found that the scale and the time course of single-subject discrimination were in relatively good agreement with their group-based counterpart - see Fig. 3b, c for identity and gender discrimination in a representative subject.

Next, to investigate the relationship between neural-based discrimination and behavioral performance we correlated classification results with recognition accuracy across participants. This analysis found an abrupt increase at about $145 \mathrm{~ms}$ that reached significance at $321 \mathrm{~ms}$ for identity and at $430 \mathrm{~ms}$ for gender (Fig. 4) - results were corrected for multiple comparisons at a more liberal level $(q<0.10)$. Thus, while exploratory in nature, these results serve to emphasize the functional significance of the discrimination results both by generally adhering to the known discrimination time course and by favoring within-gender discrimination, in better agreement with the specifics of the behavioral task.

Figure 4 


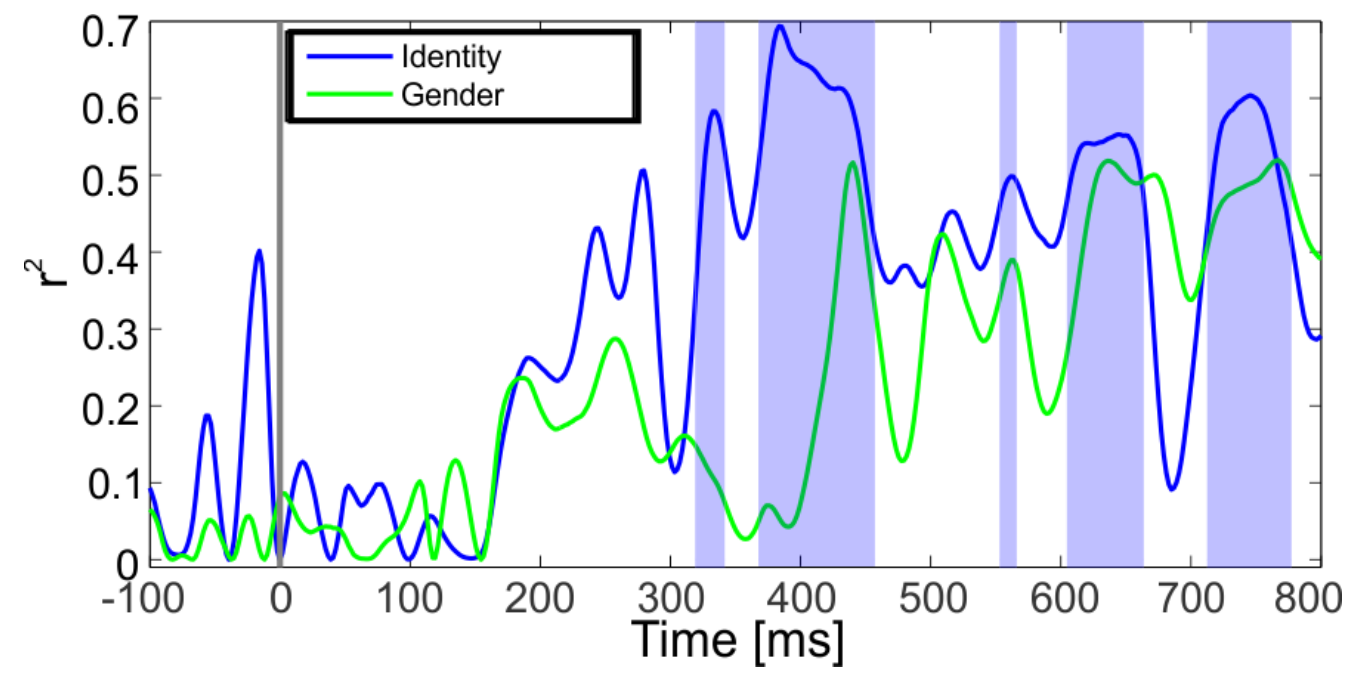

The spatial profile of identity discrimination - pattern classification

In order to clarify the topographical nature of face discrimination, SVM-based classification was applied separately to each electrode across entire epochs. This analysis revealed significant classification levels for almost all electrodes (excluding FT7, FP1, F8 and FT8 for identity and FT8 for gender) with discrimination peaks at OT sites (Fig. 5).

Figure 5
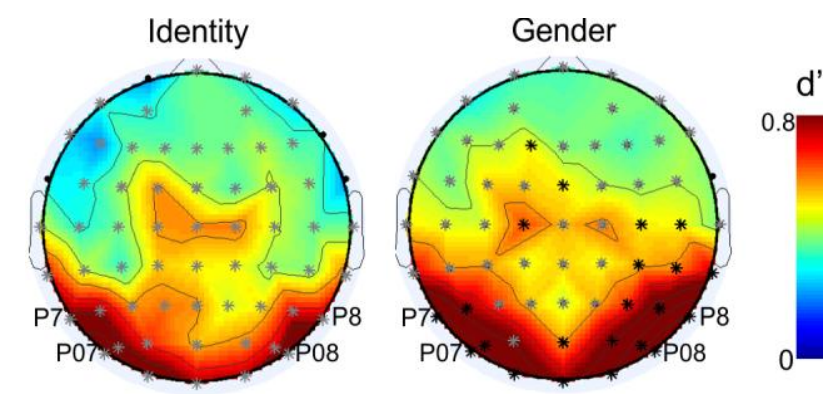

Further, a more detailed investigation considered consecutive, non-overlapping 50mswindows rather than entire epochs. The results of this investigation are consistent with early discrimination effects (between 100-150 ms) and display a systematic progression from posterior to anterior regions across consecutive time intervals (see Fig.6). 
Figure 6

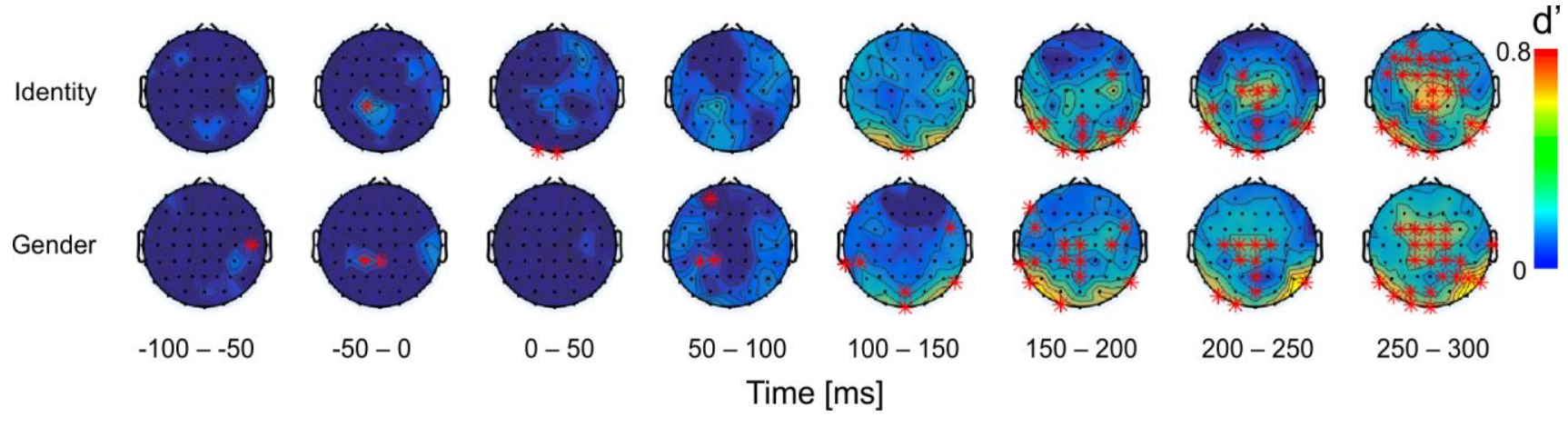

\section{Discussion}

The current work examines the temporal profile of identity discrimination as revealed by the application of pattern classification to ERP signals. Our findings suggest extensive sensitivity, with an early onset, to identity-related information. This sensitivity is present at the location of traditional components associated with face processing, such as the N170, while it extends far beyond such components both in the temporal and in the spatial domain. Overall, these results establish that ERP patterns provide a rich source of information regarding the processing of facial identity. The precise nature of this information needs to be considered though in order to clarify the mechanism for individual face recognition beyond its broad spatiotemporal profile.

Here, we investigate the neural correlates of successful face recognition (as indexed by an average accuracy higher than 90\%). To minimize the involvement of higher-level semantic processing we constrained our stimuli to images of unfamiliar individuals extracted from a 
relatively homogeneous face dataset (in terms of relative age, race etc.). Further, identity discrimination was considered separately within and across genders given that facial gender can introduce additional visual and semantic information to processing and can impact the ERP signal accordingly (Mouchetant-Rostaing and Giard, 2003). In agreement with this distinction, we found systematically higher levels of discrimination, albeit not statistically significant, for identities of different gender compared to those of the same gender. More importantly, the early onset of discrimination, especially in the context of all-electrode pattern classification, suggests a visual rather than conceptual basis for discrimination.

At the same time, the early onset mentioned above (i.e, around 70ms for both types of discrimination) is consistent with its reliance upon lower-level image properties and their processing. We note that several aspects of our study aimed to minimize the impact of global low-level properties on recognition. For instance, training and testing were conducted with different sets of images for classification purposes. Specifically, we investigated identity discrimination invariant across emotional expression. Also, stimuli were carefully processed to eliminate global differences in their appearance (e.g., due to size, contrast). However, these manipulations still leave open the contribution of local image differences to identification (e.g., the saliency of the eyebrows).

A more targeted investigation of OT electrodes, associated with the N170 component, also produced evidence for identity encoding at the time of this specific component. Interestingly, sensitivity to identity-related information was suggested by standard univariate analysis (Figs.2, Supplementary Fig. 1) and confirmed by classification-based analyses (Fig. 3, Supplementary Figs. 2, 3). Overall, these results are consistent with previous ERP work (Balas \& Saville, 2015; Caharel et al., 2009; Guillaume et al., 2009; Heisz et al., 2006; Jacques et al., 2007) suggesting the relevance of N170 for facial identity processing. 
At a general level, we note though that, in all likelihood, multiple sources of diagnostic information contribute to discrimination at different points in space and time. Thus, early ERP components, such as P1, are typically associated with category-level face processing (Dering et al., 2011) or with general-purpose low-level image processing (Dering et al., 2009; Nakashima et al., 2008; Rossion and Caharel, 2011; Rossion et al., 2003). However, evidence from related fields is consistent with an early onset of individual face recognition. For instance, single-unit recordings from middle face patch neurons in the macaque brain exhibit sensitivity to facial feature changes as early as $75 \mathrm{~ms}$ after stimulus presentation (Freiwald et al., 2009). Also, behavioral findings indicate that $75-100 \mathrm{~ms}$ of exposure to a stimulus may be sufficient for extracting identity-specific information (Lehky, 2000; Tanaka and Curran, 2001). Last, relevantly here, identity-related local information about the eyes or the mouth can be decoded, to some extent, from fMRI patterns in early visual areas (Petro et al., 2013).

On the other hand, higher-level factors are likely to contribute to discrimination at later stages. For instance, discrimination evident at frontal sites around $200 \mathrm{~ms}$ and especially after 250 ms may relate to the No-Go nature of our experimental task. Specifically, it is likely that some of these later effects reflect decision-making processes and, in particular, response inhibition, as indexed by the N2b or P3 components (Gentile \& Jansma,2012; Patel \& Azzam, 2005; Pfefferbaum, Ford, Weller, \& Kopell, 1985).

Thus, the present findings support the idea that visual information regarding facial identity can be gleaned from the ERP signal both around traditional ERP components and outside them. Confirming and extending the current findings with the use of a larger stimulus set is a necessary step for future investigations. More generally though, elucidating the precise nature of the information supporting face discrimination is critical for establishing the relevance of these findings. For instance, recent applications of reverse correlation to univariate EEG signals 
(Hansen et al., 2010; Smith et al., 2012) have been successful in uncovering the general features guiding face detection in the human cortex. The extension of this approach to individual face recognition could be instrumental in revealing the specific features involved in identity encoding along with their respective time course. In particular, exploiting the rich spatiotemporal structure of EEG patterns, as related to a sizable set of facial stimuli, could allow a closer look at the finegrained face representations underlying recognition. Arguably, the current results are particularly supportive of this direction of research.

Further, speaking to the theoretical relevance of the present findings, the correlation between ERP-based discrimination and behavioral performance provides support for joint brainbehavior accounts of visual recognition. Specifically, we found that recognition accuracy and ERP-based pattern discrimination correlated with each other across participants especially after $321 \mathrm{~ms}$ following stimulus onset. These results further validate the informational content underlying ERP-based discrimination by confirming its relevance and explanatory power regarding recognition performance.

At the same time, from a methodological perspective, these results clearly showcase the benefit of applying pattern analyses to ERP signals. This benefit is apparent both in the ability to identify intervals of significant discrimination that are not captured by conventional analyses (e.g., at early stages) as well as in the ability to boost discrimination effects at intervals captured by alternative types of analyses (Supplementary Fig. 4).

In summary, the present work investigated the temporal profile of individual face recognition as revealed by ERP patterns. Our results provide evidence for the extensive time course of facial recognition and for its early onset. Further, they suggest that identity-related information is widely distributed in both the temporal and spatial domain. Last, they underscore 
the advantage of deploying pattern analysis methods as a powerful approach to elucidating the neural mechanisms for visual face recognition. 


\section{Competing interests}

The authors declare that they have no competing interests.

\section{Acknowledgements}

This research was supported by the Natural Sciences and Engineering Research Council of Canada and by a Connaught New Researcher Award (to A.N.). Portions of the research in this paper use the FERET database of facial images collected under the FERET program, sponsored by the DOD Counterdrug Technology Development Program Office. 


\section{References}

Anzellotti, S., Fairhall, S.L., Caramazza, A., 2014. Decoding representations of face identity that are tolerant to rotation. Cereb. Cortex 24, 1988-1995. doi:10.1093/cercor/bht046

Balas, B., Saville, A., 2015. N170 face specificity and face memory depend on hometown size. Neuropsychologia 69, 211-217. doi: 10.1016/j.neuropsychologia.2015.02.005.

Benjamini, Y., Hochberg, Y., 1995. Controlling the False Discovery Rate: A Practical and Powerful Approach to Multiple Testing. J. R. Stat. Soc. Ser. B 57, 289 - 300. doi:10.2307/2346101

Bentin, S., Allison, T., Puce, A., Perez, E., McCarthy, G., 1996. Electrophysiological studies of face perception in humans. J. Cogn. Neurosci. 8, 551-565. doi:10.1162/jocn.1996.8.6.551

Bentin, S., Deouell, L.Y., 2000. Structural encoding and identification in face processing: ERP evidence for separate mechanisms. Cogn. Neuropsychol. 17, 35-54.

Bentin, S., Taylor, M.J., Rousselet, G.A., Itier, R.J., Caldara, R., Schyns, P.G., Jacques, C., Rossion, B., 2007. Controlling interstimulus perceptual variance does not abolish N170 face sensitivity. Nat. Neurosci. 10, 801-802. doi:10.1038/nn0707-801

Bishop, C.M., 2006. Pattern Recognition and Machine Learning (Information Science and Statistics). Springer - Verlag New York . doi:10.1117/1.2819119

Brainard, D.H., 1997. The Psychophysics Toolbox. Spat. Vis. 10, 433-436. doi:10.1163/156856897X00357

Caharel, S., D’Arripe, O., Ramon, M., Jacques, C., Rossion, B., 2009. Early adaptation to repeated unfamiliar faces across viewpoint changes in the right hemisphere: evidence from 
the N170 ERP component. Neuropsychologia 47, 639-43.

doi:10.1016/j.neuropsychologia.2008.11.016

Causeur, D., Chu, M.-C., Hsieh, S., Sheu, C.-F., 2012. A factor-adjusted multiple testing procedure for ERP data analysis. Behav. Res. Methods 44, 635-643. doi:10.3758/s13428012-0230-0

Chang, C.-C., Lin, C.-J., 2011. LIBSVM. ACM Trans. Intell. Syst. Technol. 2, 1-27. doi:10.1145/1961189.1961199

Debruille, J. B., Guillem, F., Renault, B., 1998. ERPs and chronometry of face recognition: Following-up Seeck et al. and George et al. Neuroreport, 9(15), 3349-3353.

Dering, B., Martin, C.D., Moro, S., Pegna, A.J., Thierry, G., 2011. Face-Sensitive Processes One Hundred Milliseconds after Picture Onset. Front. Hum. Neurosci. 5, 1-14. doi:10.3389/fnhum.2011.00093

Dering, B., Martin, C.D., Thierry, G., 2009. Is the N170 peak of visual event-related brain potentials car-selective? Neuroreport 20, 902-6. doi:10.1097/WNR.0b013e328327201d

Eimer, M., 2011. The face-sensitive N170 component of the event-related brain potential, in: The Oxford Handbook of Face Perception. pp. 329-344. doi:10.1093/oxfordhb/9780199559053.013.0017

Freiwald, W.A., Tsao, D.Y., Livingstone, M.S., 2009. A face feature space in the macaque temporal lobe. Nat. Neurosci. 12, 1187-1196. doi:10.1038/nn.2363

Gentile, F., Jansma, B. M., 2012. Temporal dynamics of face selection mechanism in the context of similar and dissimilar faces: ERP evidence for biased competition within the ventral 
occipito-temporal cortex using ICA. NeuroImage, 59(1), 682-694.

http://doi.org/10.1016/j.neuroimage.2011.07.018

Goesaert, E., Op de Beeck, H.P., 2013. Representations of facial identity information in the ventral visual stream investigated with multivoxel pattern analyses. J. Neurosci. 33, 85498558. doi:10.1523/JNEUROSCI.1829-12.2013

Guillaume, C., Guillery-Girard, B., Chaby, L., Lebreton, K., Hugueville, L., Eustache, F., Fiori, N., 2009. The time course of repetition effects for familiar faces and objects: An ERP study. Brain Res. 1248, 149-161.

Halgren, E., Raij, T., Marinkovic, K., Jousmäki, V., Hari, R., 2000. Cognitive Response Profile of the Human Fusiform Face Area as Determined by MEG. Cereb. Cortex 10, $69-81$. doi:10.1093/cercor/10.1.69

Hansen, B.C., Thompson, B., Hess, R.F., Ellemberg, D., 2010. Extracting the internal representation of faces from human brain activity: an analogue to reverse correlation. Neuroimage 51, 373-390. doi:10.1016/j.neuroimage.2010.02.021

Harris, A.M., Nakayama, K., 2008. Rapid adaptation of the $\mathrm{m} 170$ response: importance of face parts. Cereb. cortex 18, 467-476. doi:10.1093/cercor/bhm078

Haxby, J. V, Gobbini, M.I., Furey, M.L., Ishai, A., Schouten, J.L., Pietrini, P., 2001. Distributed and overlapping representations of faces and objects in ventral temporal cortex. Science 293, 2425-2430. doi:10.1126/science.1063736

Heisz, J.J., Watter, S., Shedden, J.M., 2006. Automatic face identity encoding at the N170. Vision Res. 46, 4604-4614. 
Herrmann, M.J., Ehlis, A.C., Ellgring, H., Fallgatter, A.J., 2005. Early stages (P100) of face perception in humans as measured with event-related potentials (ERPs). J. Neural Transm. 112, 1073-1081. doi:10.1007/s00702-004-0250-8

Huddy, V., Schweinberger, S.R., Jentzsch, I., Burton, A.M., 2003. Matching faces for semantic information and names: an event-related brain potentials study. Cogn. Brain Res. 17, 314 326. doi:10.1016/S0926-6410(03)00131-9

Itier, R.J., Taylor, M.J., 2002. Inversion and Contrast Polarity Reversal Affect both Encoding and Recognition Processes of Unfamiliar Faces: A Repetition Study Using ERPs. Neuroimage 15, 353-372. doi:10.1006/nimg.2001.0982

Jacques, C., D’Arripe, O., Rossion, B., 2007. The time course of the inversion effect during individual face discrimination. J. Vis. 7, 1-9. doi:10.1167/7.8.3.Introduction

Jung, T.P., Makeig, S., Humphries, C., Lee, T.W., McKeown, M.J., Iragui, V., Sejnowski, T.J., 2000. Removing electroencephalographic artifacts by blind source separation. Psychophysiology 37, 163-78.

Kriegeskorte, N., Formisano, E., Sorger, B., Goebel, R., 2007. Individual faces elicit distinct response patterns in human anterior temporal cortex. Proc. Natl. Acad. Sci. U. S. A. 104, 20600-20605. doi:10.1073/pnas.0705654104

Kuefner, D., de Heering, A., Jacques, C., Palmero-Soler, E., Rossion, B., 2010. Early Visually Evoked Electrophysiological Responses Over the Human Brain (P1, N170) Show Stable Patterns of Face-Sensitivity from 4 years to Adulthood. Front. Hum. Neurosci. 3, 67. doi:10.3389/neuro.09.067.2009 
Langner, O., Dotsch, R., Bijlstra, G., Wigboldus, D.H.J., Hawk, S.T., van Knippenberg, A., 2010. Presentation and validation of the Radboud Faces Database. Cogn. Emot. 24, 1377-1388. doi:10.1080/02699930903485076

Lehky, S. R. (2000). Fine discrimination of faces can be performed rapidly. Journal of Cognitive Neuroscience, 12(5), 848-55. http://doi.org/10.1162/089892900562453

Linkenkaer-Hansen, K., Palva, J.M., Sams, M., Hietanen, J.K., Aronen, H.J., Ilmoniemi, R.J., 1998. Face-selective processing in human extrastriate cortex around $120 \mathrm{~ms}$ after stimulus onset revealed by magneto- and electroencephalography. Neurosci. Lett. 253, 147-150. doi:10.1016/S0304-3940(98)00586-2

Liu, J., Harris, A., Kanwisher, N., 2002. Stages of processing in face perception: an MEG study. Nat. Neurosci. 5, 910-916. doi:10.1038/nn909

Martinez, A., Benavente, R., 1998. The AR face database. No 24.

Meng, J., Meriño, L.M., Shamlo, N.B., Makeig, S., Robbins, K., Huang, Y., 2012. Characterization and Robust Classification of EEG Signal from Image RSVP Events with Independent Time-Frequency Features. PLoS One 7. doi:10.1371/journal.pone.0044464

Misaki, M., Kim, Y., Bandettini, P.A., Kriegeskorte, N., 2010. Comparison of multivariate classifiers and response normalizations for pattern-information fMRI. Neuroimage 53, 103118. doi:10.1016/j.neuroimage.2010.05.051

Mouchetant-Rostaing, Y., Giard, M.H., 2003. Electrophysiological correlates of age and gender perception on human faces. J. Cogn. Neurosci. 15, 900-910.

doi:10.1162/089892903322370816 
Moulson, M.C., Balas, B., Nelson, C., Sinha, P., 2011. EEG correlates of categorical and graded face perception. Neuropsychologia 49, 3847-3853.

doi:10.1016/j.neuropsychologia.2011.09.046

Nakashima, T., Kaneko, K., Goto, Y., Abe, T., Mitsudo, T., Ogata, K., Makinouchi, A., Tobimatsu, S., 2008. Early ERP components differentially extract facial features: evidence for spatial frequency-and-contrast detectors. Neurosci. Res. 62, 225-235. doi:10.1016/j.neures.2008.08.009

Natu, V.S., Jiang, F., Narvekar, A., Keshvari, S., Blanz, V., O’Toole, A.J., 2010. Dissociable neural patterns of facial identity across changes in viewpoint. J. Cogn. Neurosci. 22, 15701582. doi:10.1162/jocn.2009.21312

Nemrodov, D., Itier, R.J., 2012. Is the rapid adaptation paradigm too rapid? Implications for face and object processing. Neuroimage 61,812-822. doi:10.1016/j.neuroimage.2012.03.065

Nestor, A., Plaut, D.C., Behrmann, M., 2011. Unraveling the distributed neural code of facial identity through spatiotemporal pattern analysis. Proc. Natl. Acad. Sci. U. S. A. 108, 999810003.

Patel, S. H., Azzam, P. N., 2005. Characterization of N200 and P300: Selected studies of the Event-Related Potential. International Journal of Medical Sciences, 2(4), 147-154. http://doi.org/10.7150/ijms.2.147

Pelli, D.G., 1997. The VideoToolbox software for visual psychophysics: transforming numbers into movies. Spat. Vis. 10, 437-442. 
Petro, L.S., Smith, F.W., Schyns, P.G., Muckli, L., 2013. Decoding face categories in diagnostic subregions of primary visual cortex. Eur. J. Neurosci. 37, 1130-1139. doi:10.1111/ejn.12129

Pfefferbaum, A., Ford, J. M., Weller, B. J., Kopell, B. S., 1985. ERPs to response production and inhibition. Electroencephalography and Clinical Neurophysiology, 60(5), 423-34.

Phillips, P.J., Rizvi, S.A., Rauss, P.J., 2000. The FERET evaluation methodology for facerecognition algorithms. IEEE Trans. Pattern Anal. Mach. Intell. 22, 1090-1104. doi: $10.1109 / 34.879790$

Phillips, P.J., Wechsler, H., Huang, J., Rauss, P.J., 1998. The FERET database and evaluation procedure for face-recognition algorithms. Image Vis. Comput. 16, 295-306. doi:10.1016/S0262-8856(97)00070-X

Rossion, B., Caharel, S., 2011. ERP evidence for the speed of face categorization in the human brain: Disentangling the contribution of low-level visual cues from face perception. Vision Res. 51, 1297-1311. doi:10.1016/j.visres.2011.04.003

Rossion, B., Jacques, C., 2011. The N170: Understanding the Time Course of Face Perception in the Human Brain. Oxford Handb. Event-Related Potential Components 1-30. doi:10.1093/oxfordhb/9780195374148.013.0064

Rossion, B., Jacques, C., 2008. Does physical interstimulus variance account for early electrophysiological face sensitive responses in the human brain? Ten lessons on the N170. Neuroimage 39, 1959-1979. doi:10.1016/j.neuroimage.2007.10.011 
Rossion, B., Joyce, C. A, Cottrell, G.W., Tarr, M.J., 2003. Early lateralization and orientation tuning for face, word, and object processing in the visual cortex. Neuroimage 20, 16091624. doi:10.1016/j.neuroimage.2003.07.010

Rousselet, G. A., Pernet, C.R., 2011. Quantifying the time course of visual object processing using ERPs: It's time to up the game. Front. Psychol. 2, 1-6. doi:10.3389/fpsyg.2011.00107

Schweinberger, S.R., Pickering, E.C., Burton, A.M., Kaufmann, J.M., 2002. Human brain potential correlates of repetition priming in face and name recognition. Neuropsychologia 40, 2057-2073.

Smith, M.L., Gosselin, F., Schyns, P.G., 2012. Measuring internal representations from behavioral and brain data. Curr. Biol. 22, 191-196. doi:10.1016/j.cub.2011.11.061

Stewart, A.X., Nuthmann, A., Sanguinetti, G., 2014. Single-trial classification of EEG in a visual object task using ICA and machine learning. J. Neurosci. Methods 228, 1-14. doi:10.1016/j.jneumeth.2014.02.014

Tanaka, J., Curran, T., Porterfield, A.L., Collins, D., 2006. Activation of preexisting and acquired face representations: The N250 event-related potential as an index of face familiarity. J. Cogn. Neurosci. 18, 1488-1497.

Tanaka, J.W., Curran, T., 2001. A neural basis for expert object recognition. Psychol. Sci. a J. Am. Psychol. Soc. / APS 12, 43-47. doi:10.1111/1467-9280.00308

Zheng, X., Mondloch, C.J., Segalowitz, S.J., 2012. The timing of individual face recognition in the brain. Neuropsychologia 50, 1451-1461. doi:10.1016/j.neuropsychologia.2012.02.030 


\section{Figure captions}

Figure 1. Experimental face stimuli.

Figure 2. Mean amplitudes of three ERP components (a- P1, b- N170, c- N250) in response to four facial identities. Results are shown separately for the right and left hemisphere at specific OT sites (error bars show $\pm 1 \mathrm{SE}$ ).

Figure 3. ERP-based identity discrimination for faces of the same gender (blue) or of different genders (green). Discrimination levels are based on classification performance as applied to patterns of ERP amplitudes across all electrodes. The horizontal axis marks time relative to stimulus onset. Results are shown (a) at the group level across all participants and (b, c) in a single participant. Shaded areas designate time points displaying above-chance withingender discrimination (two-tailed t-test, $\mathrm{q}<0.05$ ) for group analyses and within/cross-gender discrimination for single-subject analyses (permutation test, $\mathrm{q}<0.05$ ). Chance performance (solid red line) and $95 \%$ confidence intervals (dashed red lines) for single-subject analyses are based on a total of $10^{4}$ permutations.

Figure 4. Correlation between ERP-based discrimination and behavioral accuracy across participants. Shaded areas designate periods with significant correlation between within-gender discrimination and behavioral performance (Pearson correlation; $\mathrm{q}<0.10$ ). A boost in correlation values occurs around $170 \mathrm{~ms}$ and it reaches significance at $326 \mathrm{~ms}$ after stimulus onset.

Figure 5. ERP-based identity discrimination for faces of the same gender (left) or of different genders (right). Discrimination levels are based on classification performance as applied to temporal patterns of ERP amplitudes (from 0 to $800 \mathrm{~ms}$ ) separately for each electrode (twotailed t-test; $* q<0.05 ; * q<0.001)$. While the sites most efficient at identity discrimination 
correspond to the classical occipital and OT sites involved in visual processing, significant discrimination levels can be found at additional sites.

Figure 6. ERP-based identity discrimination for faces of the same gender (top) or of different genders (bottom). Discrimination levels are based on classification performance as applied to temporal patterns of ERP amplitudes (over successive windows of $50 \mathrm{~ms}$ ) separately for each electrode. Red stars mark above-chance discrimination (two-tailed t-test; $q<0.05$ ). Significant discrimination levels are present at occipital and OT sites at around $100 \mathrm{~ms}$ after stimulus presentation and then spread from posterior to anterior regions from 150 to $300 \mathrm{~ms}$. 